\title{
Turner syndrome: the patients' view
}

\author{
Lígia Z. C. Suzigan, ${ }^{1}$ Roberto B. Paiva e Silva, ${ }^{2}$ Sofia H. V. Lemos Marini, ${ }^{3}$ \\ Maria Tereza M. Baptista, ${ }^{4}$ Gil Guerra Jr., ${ }^{5}$ Luís Alberto Magna, ${ }^{6}$ Andréa T. Maciel Guerra ${ }^{6}$
}

\begin{abstract}
Objective: To identify how patients with Turner syndrome perceive their condition.

Methods: Thirty-six women with Turner syndrome, aged between 15 and 25 years and with over 2 years of medical follow-up, were individually interviewed about: the impact of Turner syndrome at the moment of the diagnosis, their understanding of the syndrome, its effect in their lives, and their expectations for the future.

Results: Only $31 \%$ of the patients immediately understood the diagnosis. Their feelings associated to that moment were neutral $(47 \%)$ or concerned $(33 \%)$. About one third of the interviewed women were unable to explain the etiology of Turner syndrome ( $42 \%)$, did not relate their symptoms with Turner syndrome (36\%), and/or believe there might be a cure for it $(44 \%)$. Although most of the interviewed women affirm that the syndrome has no interference in their lives (67\%) and that they consider themselves happy persons (78\%), in more than half of the interviews there are evidences of difficulties in social interaction and love relationship, low self-esteem, dissatisfaction with their physical appearances, especially the short stature and infertility. Their hopes for the future refer mainly to study and have a job. Although being, on average, 19 years old, one in two women (53\%) still hopes to grow up.

Conclusion: Besides medical treatment, it is important that the knowledge of the patients about the syndrome and some issues as infertility, short stature, self-esteem and social interactions receive proper and continuous attention from the moment of the diagnosis. The ideal situation should be a joint-action of a psychologist and the medical team.
\end{abstract}

J Pediatr (Rio J). 2004;80(4):309-14: Turner syndrome, sex chromosomes aberration, psychosocial aspects, patient's view, emotional impact.

\section{Introduction}

Turner syndrome (TS) occurs in approximately 1 in 2,130 female live births ${ }^{1}$ and results from the presence of complete or partial X monosomy. The clinical manifestations of TS are short stature ${ }^{2}$ - the final height ranges, on

1. Psychologist; M.Sc., Department of Pediatrics, School of Medicine, Universidade Estadual de Campinas (UNICAMP), Campinas, SP, Brazil.

2. Ph.D.; Professor, School of Medicine, Universidade Estadual de Campinas (UNICAMP), Campinas, SP, Brazil.

3. Ph.D.; Assistant professor, Department of Pediatrics, School of Medicine, Universidade Estadual de Campinas (UNICAMP), Campinas, SP.

4. Ph.D.; Assistant professor, Department of Medical Practice, School of Medicine, Universidade Estadual de Campinas (UNICAMP), Campinas, SP, Brazil.

5. Ph.D.; Associate professor, Department of Pediatrics, School of Medicine, Universidade Estadual de Campinas (UNICAMP), Campinas, SP, Brazil.

6. Ph.D.; Full professor, Department of Medical Genetics, School of Medicine, Universidade Estadual de Campinas (UNICAMP), Campinas, SP. Financial support: FAPESP - Fundação de Amparo à Pesquisa do Estado de São Paulo (01/11977-8).

Manuscript received Nov 172003, accepted for publication May 262004. average, from 142 and $146.8 \mathrm{~cm}$, but it may vary according to parental height ${ }^{3,4}$ - and gonadal dysgenesis, which leads to primary amenorrhea, delayed pubertal development and infertility. ${ }^{5}$ Some congenital and acquired anomalies can also be observed, such as cardiovascular and renal disorders, hearing impairment, hypertension, thyroid disorders, osteoporosis and obesity, among others. A wide variety of dysmorphic signs are observed: short and/or webbed neck, broad and shield chest, cubitus valgus, low posterior hairline, prominent and low-set ears, among others. ${ }^{2}$

It is believed that the presence of so many signs and symptoms and their intensity may cause severe impairment to the psychological and social functioning of TS patients, due to the reaction of the patients or of other people to these characteristics.

Studies indicate that there is an increased risk of emotional and behavioral disorders, such as problems with social interaction and love relationships; immaturity; attention deficit/hyperactivity disorder; specific learning 
disabilities; social withdrawal; aggressive behavior; and low self-esteem. ${ }^{6-9}$

Some authors regard short stature as the major factor for emotional impact and as the reason for many psychosocial problems. ${ }^{10-14}$ Other authors believe that the association between short stature and delayed maturation is accountable for a significant proportion of these problems, ${ }^{7,15}$ in addition to the interaction of several factors in the etiology of psychosocial problems associated with TS.

Studies demonstrate low self-esteem and impaired social competence in girls with TS comparatively to short girls with a normal karyotype. ${ }^{15,16}$ After the onset of adolescence most girls feel lonely and rejected by their peers. ${ }^{17}$ Moreover, they often complain about their physical appearance. ${ }^{16}$ In some studies, patients admit that they have social problems $7,8,17$ and say that they are taunted by their peers and that they do not have close friends. ${ }^{8,9}$ In adulthood, these patients tend to lead a restricted social life. ${ }^{18,19}$

Quite often, parents overprotect these children, treating them according to their height and not according to their age, thus increasing their dependence and immaturity. 20,21 In comparison with the population at large, women with TS usually have their first sexual experience much later, are sexually less active and tend not to get married. 6,18,19,22-27

Infertility also has an enormous emotional impact and may interfere with the sexuality and self-esteem of these patients. ${ }^{28}$ As a matter of fact, older patients consider infertility to be the worst problem they have to deal with in TS. 26,29

TS patients also need to be followed up by a physician and have to undergo (some invasive) exams throughout their lives. It is common knowledge that a "chronic disease" can also cause great emotional impact.

In view of the relevance of the problems described above, the aim of the present study was to evaluate the perception of TS patients in our setting about their medical condition and the factors associated with this syndrome that cause a stronger emotional impact, so as to develop a specific psychological care program and improve the followup of these patients.

\section{Patients and methods}

TS patients followed up at the Outpatient Clinic of Pediatric Endocrinology of Hospital das Clínicas of Universidade Estadual de Campinas (UNICAMP) were interviewed. This outpatient clinic currently attends to nearly 80 patients, who are followed up on a six-month basis; the patients are treated by resident doctors directly supervised by SHVLM, GGJ and MTMB, co-authors of the present study, who have been in charge of the clinic for 20 years. This way they can standardize the clinical investigation, therapies, and attitude towards patients and their families. Thirty-seven patients met the inclusion criteria: minimum age of 15 years and minimum followup of two years. These patients were selected because they had better knowledge about the disease and medical procedures. Only one patient refused to participate in the study. The study protocol was approved by the local Research Ethics Committee, and all patients (and legal guardians) signed an informed consent form.

Ages ranged from 15 to 25 years (mean 19.9 years). Age at diagnosis ranged between 0 and 18.1 years (mean 10.6 years) and the follow-up period varied from 2 to 19.6 years (mean 9.5 years). Karyotype 45, $\mathrm{X}$ was observed in 16 patients, sex chromosome mosaicism without structural aberrations (4 45,X/46,XX, 1 45,X/46,XY, $145, X / 47, X Y Y$ ) in six, and structural aberrations of sex chromosomes with or without $45, x$ cell line mosaicism were detected in 14 patients (seven cases with an isochromosome for the long arm of the $X$ chromosome, four cases of marker chromosomes, and three cases of ring $X$ chromosomes. Twenty-two patients attended or had finished elementary school, 10 attended or had finished high school, and four were in college.

A questionnaire had to be devised for data collection, following pre-established guidelines. The questionnaire included closed and open-ended questions, as no standardized questionnaires are available for this population that allow assessing specific aspects of TS, which were the aim of this study: improved knowledge about the syndrome, about the reaction at the time of diagnosis, about the impact on current life, and about future expectations.

A pilot study was carried out with 10 patients (left out of the sample as they did not fully meet the inclusion criteria) in order to check whether the questionnaire was suitable for collection of the intended information. After that, patients were interviewed individually.

The data were typed into a database using Microsoft Excel ${ }^{\circledR}$, and later logged into the SPSS for Windows ${ }^{\circledR}$, version 10.0, for statistical analysis.

A descriptive analysis was made, followed by the comparison of proportions in order to check the effect of the level of education on the answers using the chi-square test with a significance level of 0.05 . The effect of age, age at diagnosis, and length of follow-up on the answers was also checked using the Mann-Whitney test (two groups) or the Kruskal-Wallis test (other two groups).

\section{Results}

The results are shown in Table 1.

Most patients said they had not understood the diagnosis of TS or that they could only understand it after some time. Although some patients said that this happened because of their young age at the time of diagnosis, most of them ( $78 \%$ ) did not complain of not receiving specific information at that time, and said that none of the information they have today about TS was necessary at that time. Even though they say that they did not feel "anything", one third of them said they felt concerned after the diagnosis. Most of them stated that their lives did not change after the diagnosis of TS; among those who believe their lives changed, most mentioned the necessity to follow a treatment and the improvements made through these changes. 
When they were invited to convey messages to patients who could be diagnosed with TS, all of them gave positive messages, encouraging treatment, comforting and/or emphasizing the possibility of leading a normal life and being like other persons.

The level of education, age, age at diagnosis and length of follow-up did not have any effect on the answers with regard to the reaction of patients at the time of diagnosis. Concerning the comprehension of the diagnosis at that time, although some of the patients who said they could not understand it at that time were actually younger than the others, the mean ages at diagnosis (no understanding: 8.87 years; immediate understanding: 11.31 years; and understanding after some time: 11.23 years) do not show a statistically significant difference (Kruskal-Wallis test: $\chi^{2}$ $=1.48 ; \mathrm{DF}=2 ; \mathrm{p}=0.477$ ).

Although most patients mentioned TS as the reason for medical follow-up, $22 \%$ mentioned only some type of treatment for health problems. In addition, 36\% believe their signs and symptoms are isolated problems instead of sharing a common etiology with TS.

In $42 \%$ of the cases, the patients could not explain the etiology of TS. On top of that, a significant number of patients did not mention the major signs of TS (short stature

Table 1 - Results of the interviews performed with 36 patients with Turner Syndrome (TS)

Impact at the moment of the diagnosis

Did not understand the diagnosis of TS

Feelings associated with that moment

Neutral

Concern

$47 \%$

Did not notice any changes in their lives after the diagnosis

$33 \%$

$64 \%$

Understanding of TS

Reason for medical follow-up

Mentioned TS specifically

\begin{tabular}{ll} 
Mentioned general "health problems" & $78 \%$ \\
\hline
\end{tabular}

$\begin{array}{ll}\text { Could not explain the etiology of TS } & 42 \%\end{array}$

Description of symptoms of TS

Did not mention low height

$31 \%$

Did not mention hypogonadism symptoms

$69 \%$

Believe there might be a cure for TS

Did not relate TS to the symptoms presented

$44 \%$

$36 \%$

Did not mention infertility during the interview

$39 \%$

Impact of TS on patients' current life

Feelings associated with the fact of having TS

Feel "good"/"normal"

Resignation

$78 \%$

Concern

$11 \%$

Positive aspects of the treatment

Growth in height

$11 \%$

Improvement in the quality of life

$47 \%$

Induction of pubertal development

$42 \%$

$14 \%$

Better understanding of TS

$11 \%$

Possibility of menstruating

$8 \%$

Mentioned negative aspects of the treatment $\quad 56 \%$

To undergo invasive exams/procedures $\quad 28 \%$

Frequent medical appointments $19 \%$

Did not worry about any signals/symptoms of TS $\quad 36 \%$

$\begin{array}{ll}\text { Are concerned about infertility } & 25 \%\end{array}$

Are concerned about low height $\quad 14 \%$

Believe that TS does not interfere with their lives $\quad 67 \%$

Reported only individual leisure activities $44 \%$

Do not have difficulties to make and keep friends $\quad 64 \%$

Would like to have more friends $\quad 58 \%$

Positive perception about their school and/or professional lives $64 \%$

Do not have any love relationship $\quad 83 \%$

Do not have social interaction problems $64 \%$

$\begin{array}{ll}\text { Considered themselves happy } & 78 \%\end{array}$

Expectations about the future

Still have hopes of growing in height $\quad 53 \%$

Wanted to develop secondary sexual characteristics $28 \%$ 
and signs related to hypogonadism) when they had to describe the signs of this syndrome.

When asked about the possibility of cure for TS, $44 \%$ gave an affirmative answer. The expectation of cure was more frequent among patients who had finished elementary school $(12 / 22$ or $55 \%)$ than among those who had finished high school (4/10 or $40 \%$ ), and was not mentioned at all by any of the four patients who were in college. These differences are statistically significant $\left(\chi^{2}=11.02 ; D F=4 ; p=0.026\right)$. No other effect of the level of education, age, age at diagnosis and length of follow-up was observed on the pattern of answers.

Most patients said they felt "good" or "normal" about having TS. They mentioned several positive aspects of the treatment, especially growth in height and improvement in the quality of life. The negative aspects concerned invasive procedures/exams and the necessity of frequent medical appointments.

Even though one third of the patients said they did not worry specifically about any signs of TS, some of them admitted they were worried about infertility or short stature; the least frequent concerns were with delayed pubertal development or amenorrhea ( $8 \%)$, thyroid disorders $(8 \%)$ or osteoporosis $(8 \%)$. According to one third of the patients, TS interferes with their lives, especially with their social and professional lives (problems with social interaction and with getting a job) or by bringing them problems related to infertility.

In their daily routines, individual activities (e.g.: watching TV or doing household chores) and school and/or job activities predominate. Only $22 \%$ mentioned collective or group activities (e.g.: going to church, talking to friends, visiting relatives). Individual leisure activities (e.g.: reading, watching TV and listening to music) predominate over the collective ones.

Most patients said they would like to have a bigger circle of friends; however, only $36 \%$ admitted they had difficulty in making new friends or keeping the old ones, as a consequence of shyness or bashfulness.

Despite the fact that most patients showed a positive perception about their school and/or professional lives, $22 \%$ do not study or work and $14 \%$ complained of social interaction problems. Seventy-five percent mentioned having a good relationship with colleagues, teachers/ professors and/or boss, while $47 \%$ mentioned having a good family relationship.

Out of 36 interviewed patients, 30 said they did not have any love relationship. When asked about the reason, most of them declared they had not found the ideal partner yet $(40 \%)$ and $37 \%$ said they did not feel like having a romance with anyone. For $13 \%$ of the patients this was related to their behaviors (especially shyness) while for $10 \%$ it was related to their physical appearance (especially because they did not feel pretty/attractive, and due to their short stature).

Seventeen patients ( $47 \%$ ) said they would have a better social interaction if they did not have TS, especially because they would have a better self-esteem (47\%), and also because they would have a boyfriend (12\%) and more friends (12\%).

When asked to describe themselves, $75 \%$ of the patients mentioned positive personality characteristics (e.g.: being cheerful, helpful, understanding), 47\% mentioned negative characteristics (e.g.: being "boring", shy, anxious and "edgy") and $28 \%$ mentioned negative physical characteristics (e.g.: being "fat" and "short"). Twenty-three patients (64\%) would like to change some characteristics, especially physical ones (mainly stature and weight).

Most patients considered themselves happy; $6 \%$ did not $\mathrm{know} /$ refused to answer the question. Among those who do not see themselves as happy, most mentioned the fact that they did not have a boyfriend or had TS. The level of education, age, age at diagnosis and length of follow-up did not have any effect on the answers with regard to the current impact of TS on the patients' lives.

Over $50 \%$ of the patients still hope they will grow in height, and a little bit more than one fourth of them hope to develop secondary sexual characteristics; $14 \%$ want to be "normal", $11 \%$ want to menstruate, $8 \%$ want to "improve", 6\% want to have children, $6 \%$ want to understand TS better. Their expectations about the future are mainly concerned with work $(67 \%)$ and with the possibility of furthering their studies ( $64 \%)$, in addition to getting married (39\%), having children (14\%) and/or "making a family" (14\%).

The mean age of the 19 patients (53\%) who said they still have hopes of growing in height was 19.1 years, significantly lower than those who did not have this expectation (20.92 years) (Mann-Whitney test: $p=0.026$ ). The mean age of the 10 patients ( $28 \%)$ who mentioned they wanted to develop secondary sexual characteristics (19.5 years) did not differ from those patients who did not have this expectation (20.7 years) (Mann-Whitney test: $p=$ 0.658). The level of education, age at diagnosis and length of follow-up did not have any effect on the pattern of answers related to the future expectations of the patients.

\section{Discussion}

In comparison with other multiple congenital anomalies, TS patients present with several physical problems (short stature, hypogonadism and consequent infertility, obesity, malformations, and dysmorphisms), need continuous medical follow-up, and have normal intelligence - which allows them to be aware of their limitations and of the criticism of other people. Although there should be individual variability, these aspects certainly determine an increased risk of psychological and social problems, and it is important that the magnitude of these problems be examined from the patients' own perspectives.

Despite the limitations of an individual interview, the results obtained in the present study indicate that, similarly to what has been observed in other studies, $6-8,16,17,26,29$ the patients show difficulty having social interaction and love relationships, are dissatisfied with their physical 
appearance (especially short stature), have low self-esteem, and show concern with infertility.

Concerning the indifference towards the diagnosis of TS, we should question to what extent this may be related to young age at diagnosis, made on average at 10.6 years (which would be compatible with the fact that many patients had not understood the diagnosis at the moment it was established) or whether there could have been some denial or inhibition about exposing their feelings to the interviewer, as occurred with other answers. The fact that the diagnosis did not bring any changes to the lives of $64 \%$ of the patients is somewhat curious, since these patients had to undergo several exams and had regular medical appointments, were diagnosed with associated anomalies that had gone undetected until then, in addition to having to come to terms with the possibility of being infertile. Anyway, the positive messages they would convey to patients recently diagnosed with TS show that they have apparently accepted or adapted to the situation.

Although most of the patients said they felt "good" about having TS, when the question was phrased in a different way, contradictions became evident: two thirds showed specific concerns, especially with infertility or hypogonadism; approximately half of them mentioned negative aspects about having regular medical follow-ups; most of them described themselves using personal characteristics instead of physical ones, which may reveal some difficulty in talking about their appearance; $42 \%$ would like to change their physical characteristics, mainly stature and weight; and although many of them were in late adolescence and early adulthood, they still hoped to grow in height or develop secondary sexual characteristics.

Limitations on social interaction, as observed by other authors, 18,19 are blatantly obvious when there is a predominance of individual activities and an urge to have a bigger circle of friends. Furthermore, most of the patients do not have a love relationship, and almost half of them believe that they would not have social interaction problems if they did not have TS.

Therefore, it is crucial to help patients to identify their social interaction difficulties and to teach them how to tackle them so that they can have social and love relationships that are satisfactory to them. It is also important to advise families not to overprotect their children, which increases their dependence and immaturity, but to treat them as normal individuals, thus minimizing their psychosocial difficulties. Psychologists and all the health care professionals involved should help families and patients by providing them with medical information and/or psychotherapy.

Even though the information about TS has been regularly provided since the diagnosis was established and despite 9.5 years of follow-up and several meetings with other TS patients on every appointment, the interviewed patients showed dissatisfactory knowledge about their medical condition, which suggests an intrinsic difficulty in understanding or accepting the disease. Thus, the level of information and knowledge should be regularly checked by the medical staff. Expectations of cure and fertility should be dealt with with the patients, as these issues, together with short stature and low self-esteem, are the most critical, in the patients' own opinion. These issues should receive special and continuous attention from the very moment of diagnosis. In our opinion, further studies are needed on the psychosocial aspects of TS in Brazil.

The results obtained here are relevant, mainly due to the paucity of studies with Brazilian patients on this area, and also because they allow more specific studies about the psychological and social aspects of TS to be carried out.

\section{References}

1. Nielsen J, Wohlert M. Chromosome abnormalities found among 34,910 newborn children: results from a 13-year incidence study in Arhus, Denmark. Hum Genet. 1991;87:81-3.

2. Lippe B. Turner Syndrome. In: Serling MA, editor. Pediatric Endocrinology. Philadelphia: W. B. Saunders Co., 1996. p. 387-421.

3. Hall JG, Gilchrist DM. Turner syndrome and its variants. Pediatr Clin North Am. 1990;37:1421-40.

4. Massa GG, Vanderschueren-Lodeweyckx M. Age and height at diagnosis in Turner syndrome: influence of paternal height. Pediatrics. 1991;88:1148-52.

5. Pasquino AM, Passeri F, Pucarelli I, Segni M, Municchi G. Spontaneous pubertal development in Turner's syndrome. Italian study group for Turner's syndrome. J Clin Endocrinol Metab. 1997; 82:1810-3.

6. Nielsen J, Nyborg H, Dahl G. Acta Jutlandica XLV Medicine Series 21; Arhus, Denmark, 1977.

7. Skuse D, Percy E, Stevenson J. Psychosocial functioning in the Turner syndrome: a national survey. In: Stabler B, Underwood L, editors. Growth, Stature and Adaptation. Behavioral, Social and Cognitive Aspects of Growth Delay. Chapel Hill: The University of North Carolina; 1994. p. 15-64.

8. Boman UW, Möller A, Albertsson-Wikland K. Self-perception, behavior and social functioning in Swedish girls with Turner syndrome: a population-based study. Göteborg Psychological Reports; Göteborg University Sweden. 2000;30(5):1-12.

9. McCauley E, Feuillan P, Kushner H, Ross JL. Psychosocial development in adolescents with Turner syndrome. J Dev Behav Pediatr. 2001;22:360-5.

10. Brinch M, Manthorpe T. Short stature as a possible etiological factor in anorexia nervosa. Acta Psychiatr Scand. 1987;76: 328-32.

11. Massa GG, Vanderschueren-Lodeweyckx M, Malvaux P. Linear growth in patients with Turner Syndrome: influence of spontaneous puberty and parental height. Eur J Pediatr. 1990;149:246-50.

12. Huisman J, Slijper FME, Sinnema G, Akkerhuis GW, BrugmanBoezeman ATM, Feenstra J, et al. Psychosocial functioning and effects of growth hormone treatment in Turner syndrome. In: Hibi I, Takano K, editors. Basic and clinical approach to Turner syndrome. Amsterdam: Elsevier Science Publishers; 1993. p. 157-61.

13. Wilson DM, McCauley E, Brown DR, Dudley R. Oxandrolone therapy in constitutionally delayed growth and puberty. Pediatrics. 1995;96:1095-100.

14. Gilmour J, Skuse D. Short stature - the role of intelligence in psychosocial adjustment. Arch Dis Child. 1996;75:25-31.

15. McCauley E, Ito J, Kay T. Psychosocial functioning in girls with Turner's syndrome and short stature: social skills, behavior problems, and self-concept. J Am Acad Child Psychiatry. $1986 ; 25: 105-12$.

16. McCauley E, Ross JL, Kushner H, Cutler G Jr. Self-esteem and behavior in girls with Turner syndrome. J Dev Behav Pediatr. $1995 ; 16: 82-8$.

17. Swillen A, Fryns JP, Kleczkowska A, Massa G, VanderschuerenLodeweyckx M, Van den Berghe $H$. Intelligence, behavior and psychological development in Turner syndrome. Genet Couns. $1993 ; 4: 7-18$ 
18. McCauley E, Sybert V, Ehrhardt AA. Psychosocial adjustment of adult women with Turner syndrome. Clin Genet. 1986;29:284-90.

19. Aran O, Galatzer A, Kauli R, Nagelberg N, Robicsek Y, Laron Z. Social, educational and vocational status of 48 young adult females with gonodal dysgenesis. Clin Endocrinol (Oxf). 1992;36:405-10.

20. Nielsen J, Sillesen I. Turner's syndrome in 115 Danish girls born between 1955 and 1966. Acta Jutlandica LIV Medicine Series 22. Aarhus, Denmark; 1981.

21. Nielsen J. What more can be done for girls and women with Turner syndrome? In: Hibi I, Takano K, editors. Basical and clinical approach to Turner syndrome. Amsterdam: Excerpta Medica; 1993. p. 169-76.

22. Downey J, Ehrhardt AA, Gruen R, Bell JJ, Morishima A. Psychopatology and social functioning in women with Turner syndrome. J Nerv Ment Dis. 1989;177:191-201.

23. Pavlidis K, McCauley E, Sybert V. Psychosocial and sexual functioning in women with Turner syndrome. Clin Genet. 1995; 47:85-9.

24. Berch D, McCauley E. Psychosocial functioning of individuals with sex chromosome abnormalities. In: Holmes $C$, editor. Psychoneuroendocrinology. Brain, Behavior and Hormonal Interactions. New York: Springer-Verlag; 1990. p. 164-83.

25. Raboch J, Kobilkova J, Horejsi J, Starka L, Raboch J. Sexual development and life of women with gonodal dysgenesis. J Sex Marital Ther. 1987;13:117-27.
26. Tang G. Bio-psycho-social aspects of gonodal dysgenesis. J Psychosom Obstet Gynecol. 1989;10:113-9.

27. Holl RW, Kunze D, Etzrodt $H$, Teller W, Heinze E. Turner syndrome: final height, glucose tolerance, bone density and psychosocial status in 25 adult patients. Eur J Pediatr. 1994; 153:11-16.

28. Tesch LG. Benefits of support groups for those affected by Turner Syndrome and the associated medical community. In: Hibi I, Takano K, editors. Basic and clinical approach to Turner syndrome. Amsterdam: Elsevier Science Publishers; 1993. p. 185-92.

29. Sylven L, Magnusson C, Hagenfeldt K, von Schoultz B. Life with Turner syndrome: a psychosocial report from 22 middle-aged women. Acta Endocrinol (Copenh). 1993;129:188-94.

Corresponding author:

Andréa Trevas Maciel-Guerra

Departamento de Genética Médica - FCM - UNICAMP

Caixa Postal 6111

CEP 13083-970 - Campinas, SP, Brazil

Tel.: +55 (19) 3788.8907

E-mail: atmg@fcm.unicamp.br 